\title{
Bioenergy Decision Support Systems: Worth the Effort?
}

\author{
Daniel Wright ${ }^{1, *}$, Prasanta Dey ${ }^{1}$, John Brammer $^{1}$, Phil Hunt ${ }^{2}$ \\ ${ }^{1}$ Aston University, Birmingham, UK \\ ${ }^{2}$ Enco Energy Limited, UK \\ *E-mail:Wrightd1@Aston.ac.uk
}

\begin{abstract}
The purpose of this research is to explore the disparity between the existing model-orientated bioenergy decision support system (DSS) functions and what is desired by practitioners, in particular bioenergy project developers. This research has compiled the published bioenergy project development models, to highlight the characteristics emphasised by academics. When contrasted against a UK practitioner's perspective through the administration of a Likert style questionnaire, it is clear that the general DSS issues still persist. Finally, the research suggests how this 'theory-practice' divide could be addressed. The research contributes by giving a unique insight into the demands of a practitioner, but is currently limited by a small sample size.
\end{abstract}

Keywords: Decision Support System, Bioenergy Project Development, Theory-Practice Divide

\section{Introduction}

Developing a bioenergy project requires large volumes of complex information to be gathered and processed by project developers. This information tends to be fairly structured and accessible; although, often not easily retrievable for use in a timely manner [1]. The difficulties faced in progressing through the necessary phases in bioenergy project development encourage a dependence on experts, which restricts knowledge transfer and nonexpert project developers entering the industry - ultimately constraining sector growth. This could be one explanation why growth in the UK bioenergy market, in particular has been slower than expected, with some referring to growth as 'slight' [2].

Academic literature supports the use of model-oriented decision support systems as a proven method to aid both expert and non-expert decision-makers [3], with a substantial amount of bioenergy support systems being assembled to address a multitude of development problems in countries such as: Japan, Greece and Italy. Although, this literature rarely considers the most critical issue; the theory-practice divide in DSS research [4]. At present, there is no research to understand the lack of practical application of decision support tools in the bioenergy sector. With the dire need for bioenergy project support tools to aid decisionmaking and in turn sector growth in the UK, but with a persistent disconnect between theory and practice $[4,5]$, a lack of relevance [5] and little evidence to show that these tools are ever really adopted [6] - can a decision support system really ever have a pragmatic contribution.

\subsection{Objectives}

The research objectives are to ascertain whether bioenergy DSS are worth the effort, by:

- reviewing published bioenergy project development DSS models;

- critically comparing these models to the requirements of the industry practitioner;

- discussing how future research could be more applicable to the practitioner.

\subsection{Contribution}

The contribution of this research is two-fold: it is the first paper to analyse the characteristics of all existing model-orientated DSS papers in developing bioenergy projects; and it increases understanding of the theory-practice gap by assessing these characteristics against a practitioner's perspective. 


\section{Developments and Issues with Decision Support Systems}

Since the term decision support system' was coined by Gorry and Scott-Morton [7] when suggesting a framework for improving management information systems (MIS), there have been many developments in this discipline. One of the most successful attempts to classify the characteristics of a DSS was by Alter [8] who created a taxonomy of seven categories, which could be further condensed down to two: data-orientated or model-orientated support systems. The bioenergy DSS models reviewed in this research are all model-orientated, spanning the representation and optimisation categories in Alter's [8] classification, most support systems in use today are in these two categories [4]. Model-orientated decision support systems are typified by their user(s) and whether they integrate knowledge. This can be seen in the table below:

Table 1. DSS types [9].

\begin{tabular}{|c|c|}
\hline Type & Description \\
\hline $\begin{array}{l}\text { Personal DSS } \\
\text { (PDSS) }\end{array}$ & $\begin{array}{l}\text { Small-scale systems that are normally developed for one manager, or a } \\
\text { small number of independent managers, for one decision task. }\end{array}$ \\
\hline $\begin{array}{l}\text { Group support } \\
\text { systems (GSS) }\end{array}$ & $\begin{array}{l}\text { Decision responsibility is shared by a number of managers and a number } \\
\text { of managers need to be involved in the decision process. }\end{array}$ \\
\hline $\begin{array}{l}\text { Intelligent DSS } \\
\text { (IDSS) / } \\
\text { knowledge-based } \\
\text { DSS }\end{array}$ & $\begin{array}{l}\text { Intelligent DSS can be classed into two generations: the first involved } \\
\text { the use of rule-based expert systems and the second generation uses } \\
\text { neural networks, genetic algorithms and fuzzy logic [Turban et al., } 2005 \\
\text { cited 9]. }\end{array}$ \\
\hline $\begin{array}{l}\text { Knowledge } \\
\text { management-based } \\
\text { DSS }\end{array}$ & $\begin{array}{l}\text { Involves the combination of several areas including IT, organizational } \\
\text { behaviour, organizational structure, economics and organizational } \\
\text { strategy. }\end{array}$ \\
\hline
\end{tabular}

Table 1, also illustrates the chronological order in which the field has developed. The earlier PDSS were intended for a single decision-maker or user. As the field developed, multiple decision-makers were accounted for in the DSS design. More recently, in the last two decades researchers have built in expertise to support systems to further support decision-making.

Decision support systems have been extensively applied to a myriad of industries and problems over the past four decades, with Eom [4] reviewing over 25,000 published articles; however, he concluded theory-practice divide issues still persist. A paper by Arnott and Pervan [5] reduced the field's problems to eight issues, of which the research has selected four relating to the theory-practice divide have been selected, listed in table 2:

Table 2. DSS discipline key issues [adapted from 5].

Key Issue Comments

$\begin{aligned} & \text { Professional } \\ & \text { relevance }\end{aligned}$ Most DSS research is disconnected from practice.
Reseach

Research DSS is more dominated by positivism than general IS [information methods and systems]. Case study research is under represented. A long history of paradigms design science research could contribute methodologically to IS research.

Theoretical Around half of the papers have no explicit foundation in judgement and foundations decision-making. Much DSS research is based on a relatively old Inertia and The relatively older types of PDSS and GSS still dominate research conservatism agendas. 
The first and foremost issue is 'professional relevance', Arnott and Pervan [5] found "only $10.1 \%$ of research is regarded as having high or very high practical relevance. On the other hand, $49.2 \%$ of research was regarded as either having low practical relevance or none at all”. Two of the papers, Hirschheim and Klein [10] and Banbasat and Zmud [11], which are cited in the focal article as giving possible explanations, point toward the disconnect between these two groups; both articles focus on the researcher and academia as the cause of the divide. With Hirschheim and Klein [10], in particular, analysing the performance of the discipline from an external, management viewpoint. They show how the practitioners' expectations were not fulfilled - no matter how unrealistic. The issue with this defence is that it is bias and fails to consider, the practitioners' role in this 'disconnect'.

There are a variety of possible explanations why decision support systems fail to get adopted by practitioners. The first being given by Rizzoli and Young [12] who found that decisionmakers lack trust in a DSS even if it is proven to be effective, opting for their own often suboptimal decisions. A case in point is a study of forestry operations decision-makers in Canada, who would rather rely on their own ability than computer software's [Rooney, 1996 cited 1]. This was also found by Wierzbicki and Wessels [13:37] who discovered "the higher the level and experience of a decision-maker, the less inclined she/he is to trust in various tools and methods of decision analysis and support”. The second explanation may be as Brown and Vari [6] suggested "the practical impact of decision aids on business decisions is less easy to establish, due to the cloak of commercial secrecy..." with the successful DSS being used to achieve a competitive advantage rather than for publication.

The second issue shown in table 1 is the research method and paradigm typically applied in DSS research. Arnott and Pervan [5] found that the discipline is still overwhelmingly positivist, their conclusion to remedy this was a greater reliance on case study research, and adoption of the interpretive paradigm. This issue is inextricably linked to 'practical relevance', possibly because academia has been guilty of pursuing 'rigour over relevance'[11], and the classical hypothetico-deductive positivist paradigm is the most suitable method for achieving this rigour. Although, an interpretive case study would increase the practical relevance of a DSS publication, it is not necessarily the only method of addressing this issue. It is possible to increase relevance without requiring a paradigm shift; an experimental positivist or post-positivist DSS could increase practical relevance with the empirical testing of the model(s) through actual cases application.

The third and forth issues follow on from the preceding problems in the general discipline. Arnott and Pervan [5] classified a paper as having a theoretical foundation if there was theory cited in the research design and result interpretation, they stated that the results in general DSS research was quite alarming, after finding just under half of all papers reviewed (47.8\%) lacking a theoretical foundation. Additionally, they discovered that the older - more popular types of DSS (PDSS and GSS) performed better than other DSS types. Finally, their research found that inertia and conservatism in the discipline, even accounting for the publication lag, meant that the newer types of DSS were not being fully adopted by academia.

These four issues in the general decision support system field are still present, and have not been fully addressed over the past four decades. It is unclear who is at fault: practitioners, academia, or both. However, it is clear in the context of this research problem, that if these issues are addressed by the bioenergy industry, their use and contribution will greatly increase. 


\section{Decision Support Systems in the Bioenergy Industry}

There have been many bioenergy decision support systems created for academic papers which address different issues in developing bioenergy projects. The research has excluded a small group of sustainability DSS papers, as they are beyond the scope of this research. A timeline of all the relevant bioenergy DSS literature has been compiled in figure 1; this is a substantial number of research papers considering this is a relatively new academic field. The extent to which DSS tools have been utilised in academic journals could be attributed to the complexity of effectively making decisions in the energy sector, making it is necessary to utilise some form of support model [14].

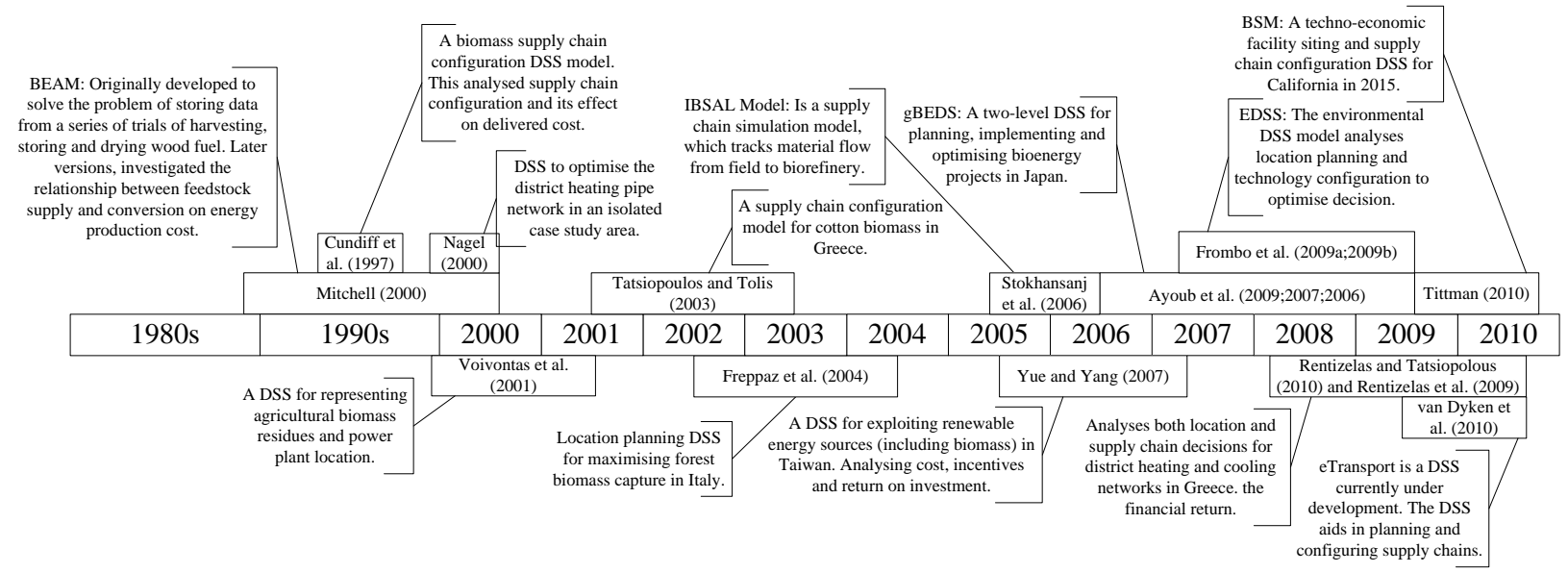

Fig. 1. Annotated timeline of model-oriented bioenergy DSS research.

The timeline highlights 13 DSS models created for developing bioenergy projects, primarily spanning two decades, with most models being created in the last 10 years. The timeline gives a brief overview of each DSS and shows the publication lag, or when explicitly stated in the article, when research began.

\section{Methodology}

The paper gives an exploratory insight into what a bioenergy project developer requires from a DSS. The primary data for this research was gathered by creating a questionnaire for practitioners, which analyses their opinion on the existing bioenergy research papers traits.

From the descriptive statistics gathered from the analysis of the DSS characteristics (table 3), a scale of importance was attributed to each characteristic. The frequency ranking scale was: low (0-2 articles); medium (3-5 articles); high (6-8 articles); very high (9 or more articles).

\subsection{Bioenergy DSS Characteristics}

Before it is possible to assess the focus of bioenergy decision support systems, it is necessary to analyse their characteristics. To achieve this key attribute categories have been created, some relate to the configuration of the DSS, such as: type of DSS, intended user(s), research paradigm etc; whereas, the remaining characteristics relate to the professional relevance and model application. The scales attributed to each of the characteristics varies, most are nonparametric nominal scales. In some cases a simple dichotic yes/no choice was utilised, as seen in table 3. 
Table 3. DSS Characteristic Classifications.

\begin{tabular}{ll}
\hline \multicolumn{1}{c}{ Category } & \multicolumn{1}{c}{ Classification } \\
\hline Type of DSS & $\begin{array}{l}\text { This characteristic refers to the DSS design. The bioenergy DSS models are } \\
\text { categorised as: personal (PDSS), group (GSS), intelligent or knowledge- } \\
\text { based, or knowledge management-based. }\end{array}$ \\
\hline User(s) & $\begin{array}{l}\text { The categories for this measure are: national or regional } \\
\text { planners/developers, local developers, investors and not stated. Importantly, } \\
\text { if the intended user of the decision support tool is not explicitly stated in the } \\
\text { research paper then they are categorised as not stated. }\end{array}$ \\
\hline Research & $\begin{array}{l}\text { The research method and paradigm categories are based on the Arnott and } \\
\text { method }\end{array}$ \\
$\begin{array}{l}\text { Pervan [5] classification: non-empirical conceptual, illustrative or applied } \\
\text { concepts; empirical objects or events and processes. }\end{array}$ \\
$\begin{array}{l}\text { Practical } \\
\text { relevance }\end{array}$ \\
$\begin{array}{l}\text { This is the foremost issue with decision support systems. As the Arnott and } \\
\text { Pexperts to judge whether the article had practical relevance (none, low, } \\
\text { medium, high, very high). It was important to implement a similar system, } \\
\text { with a less interpretive grounding. Table 4 applies a measure for each } \\
\text { existing bioenergy articles. }\end{array}$ \\
$\begin{array}{l}\text { The method of judging whether a bioenergy DSS paper has a theoretical } \\
\text { foundation will be the same as applied in Arnott and Pervan [5]. They } \\
\text { foundation }\end{array}$ \\
$\begin{array}{l}\text { distinguished between only citing other theory in the introductory chapters } \\
\text { and citing theory in the method and discussion sections, as no theoretical } \\
\text { foundation or having a theoretical foundation respectively. }\end{array}$ \\
\hline $\begin{array}{l}\text { The research also categories the DSS models by the targeted phase in the } \\
\text { bioenergy project lifecycle, the phases are: planning, construction and } \\
\text { operation [15]. As decisions are made throughout a project’s lifecycle } \\
\text { phases, it was important to ascertain where the current support systems } \\
\text { focused. }\end{array}$ \\
$\begin{array}{l}\text { The bioenergy model output(s) were also recorded as: financial, non- } \\
\text { financial or multiple. }\end{array}$ \\
\hline
\end{tabular}

As the theory-practice divide and whether decision support systems are worth the effort, is the primary focus of this research, emphasis has been placed on creating a practical relevance scale, as seen in the table below:

Table 4. Scale for establishing practical relevance.

\begin{tabular}{ll}
\hline Relevance & \\
\hline Low & Hypothetical case \\
Medium & Single application or case study \\
High & Multiple practical uses \\
Very high & Multiple practical uses and application examples \\
\hline
\end{tabular}

This research argues that a DSS which is possesses multiple practical uses; meaning it can be applied to multiple cases to solve problems (generalisable), will have the highest practical relevance. Moreover, if this is also demonstrated in the academic article, as opposed to merely stating a support system's generalisability, then this achieves 'very high' practical relevance. 


\subsection{Practitioner Questionnaire}

A simple closed choice questionnaire, with a five-point Likert style scale for importance (none, low, medium, high, very high) was designed to explore whether bioenergy project developers placed similar emphasis on the characteristics in the existing bioenergy support systems. Due to the constraints of the research paper, the questionnaire is not included, but is available on request. The results of the questionnaire can be seen in table 5 . The respondent organisation is a SME developer of biomass combined heat and power projects in the UK.

\subsection{Research Limitations}

The research is currently limited to one organisation, but this is because the research is ongoing. Although, this is presently a limited sample size, its contribution remains as the first study to explicitly state the practitioner's view.

\section{Results}

The results table (5) below, illustrates the comparisons between the academic and practitioner weighting:

Table 5. Results table.

\begin{tabular}{|c|c|c|c|}
\hline Characteristic & Application & $\begin{array}{l}\text { Academic Weighting } \\
\text { (no.) }\end{array}$ & $\begin{array}{l}\text { Practitioner } \\
\text { Weighting }\end{array}$ \\
\hline \multirow[t]{4}{*}{ Type of DSS } & PDSS & Very high (10) & High \\
\hline & GSS & Low $(0)$ & High \\
\hline & Knowledge-based & Medium (3) & Very high \\
\hline & Knowledge mgmt' based & Low (0) & Low \\
\hline \multirow[t]{4}{*}{ User(s) } & National or regional & Low (1) & Very high \\
\hline & Local & Low (0) & Very high \\
\hline & Investor & Low (1) & Very high \\
\hline & Implied/not stated & Low importance (10) & Very important \\
\hline \multirow[t]{2}{*}{ Method } & Empirical & High (7) & High \\
\hline & Non-empirical & High (6) & Low \\
\hline \multirow{2}{*}{$\begin{array}{l}\text { Practical } \\
\text { relevance }\end{array}$} & Low/med (single application) & Very high (10) & High \\
\hline & $\begin{array}{l}\text { High/v.high (multiple } \\
\text { applications) }\end{array}$ & Medium (3) & Very High \\
\hline \multirow{2}{*}{$\begin{array}{l}\text { Theoretical } \\
\text { foundation }\end{array}$} & Yes & Low (2) & Very high \\
\hline & No & Very high (11) & - \\
\hline \multirow{3}{*}{$\begin{array}{l}\text { Bioenergy } \\
\text { lifecycle phase }\end{array}$} & Planning & Very high (13) & Very high \\
\hline & Construction & Low $(0)$ & Very high \\
\hline & Operation & Low (0) & Very high \\
\hline \multirow[t]{3}{*}{ Model output } & Financial & High (7) & Very high \\
\hline & Non-financial & Low (2) & High \\
\hline & Both & Medium(4) & - \\
\hline
\end{tabular}

\subsection{Analysis}

The results highlighted that the foremost issue of practical relevance in the bioenergy sector was an improvement on the Arnott and Pervan [5] findings, with 3 models or 23.1\% classified as having a high or very high practical contribution, as opposed to only $10.1 \%$ of their sample were categorised as such. Although, it is not possible to truly know if the criteria 
for assessing this were exactly the same, this verifies the research paper's interpretation of the practical relevance scale.

Moreover, the user(s) of the bioenergy DSS tended not to be mentioned or explicitly stated (76.9\%) in the bioenergy publications, this can only support the argument for the lack of relevance, as the user is most likely the practitioner. This is something that the respondent organisation felt particular strong about, stating that it is very important to target each user group, and to explicitly tailor a DSS to their requirements. However, there is a general agreement between the academic and practitioner viewpoint on the most suitable output of the model, with the most emphasis placed on financial performance measures.

The bioenergy lifecycle phase results are also particularly interesting, as all of the 13 bioenergy models supported decision-making in the planning phase: in supply chain planning, location planning or both. None of the existing models supported the decision-maker in the later phases of a project's lifecycle; this highlights a need for further work considering that the respondent classified all phases as equally important.

The second issue raised was the lack of case study research and positivist paradigm dominance in the general DSS field. This was found to still be present in the bioenergy development models, with all papers reviewed having a positivist paradigm. Although, there was a high level of case study research found, with more than half of the bioenergy support systems possessing empirical data from case studies. This importance was also expressed in the respondent organisation's support for empirically grounded case studies.

The existing bioenergy research performed worse than the general discipline with regard to the theoretical foundation. This category classified 11 of the 13 models as not having a theoretical foundation in the model development and result analysis sections of the academic paper, this is considerably higher than the $47.8 \%$ found by Arnott and Pervan [5]. Furthermore, PDSS represented the majority of existing models, illustrating the inertia and conservatism within the discipline; although, it can be seen that group support systems were not adopted, the more recent knowledge-based DSS did quite well in this sector (23.1\%).

\section{Conclusion}

From analysing the current issues in general DSS research (section 2), it is clear that the four issues relating to the theory-practice divide are interlinked; what wasn't clear was whether the bioenergy DSS research suffered from the same shortcomings. The bioenergy article analysis and the primary research in this paper have shown that for the most part they are still present. This could, therefore, explain why there is little evidence of practical adoption.

Arguably, if the purpose of building a DSS is to support decision-making, then practical application has to be regarded as the most important parameter. The most suitable way of addressing this issue is to design a system tailored to the decision-maker - this does not appear to be a priority of the existing research. Acknowledging and explicitly integrating the requirements of the user into the model building and testing stages will undoubtedly increase adoption. Furthermore, if generalisable model-orientated support systems have the highest level of practical relevance, then where possible they should take preference over a single use model. Finally, creating knowledge-based or intelligent DSS models which allow for group decision-making will not only reduce the conservatism issue but also allow for a potentially greater contribution. Addressing the issues raised in this paper is undoubtedly the first barrier to realising the full potential of decision support systems in the bioenergy industry. 


\subsection{Further Work}

Firstly, as the research presented is at an early stage, the next step is to increase the respondent sample size to include national and regional developers and investors, to better ascertain what functionality bioenergy project developers require. Moreover, a larger sample size would allow for inferential statistical analysis, adding a greater level of depth to the research. Secondly, as there is no research into decision support models for the latter phases of a bioenergy lifecycle, this presents an interesting new avenue of enquiry.

\section{References}

[1] C. P. Mitchell, Development of decision support systems for bioenergy applications, Biomass and Bioenergy, 18, 2000, pp. 265-278.

[2] R. Slade, C. Panoutsou and A. Bauen, Reconciling bio-energy policy and delivery in the UK: Will UK policy initiatives lead to increased deployment?, Biomass and Bioenergy, 33, 2009, pp. 679-688.

[3] D. J. Power, Decision Support Systems, Greenwood Pressed, 2002.

[4] S. B. Eom, The Development of Decision Support Systems Research, The Edwin Mellen Press Ltd, 2nd ed, 2007.

[5] D. Arnott and G. Pervan, Eight key issues for the decision support systems discipline, Decision Support Systems, 44, 2008, pp. 657-672.

[6] R. Brown and A. Vari, Towards a research agenda for prescriptive decision science: The normative tempered by the descriptive, Acta Psychologica, 80, 1992, pp. 33-47.

[7] G. A. Gorry and S. S. Scott-Morton, a framework for management information systems, Sloan Management Review, 13, 1971, pp. 55 - 70.

[8] S. Alter, Decision Support Systems: Current Practice and Continuing Challenges Addison Wesley Longman ed, 1980.

[9] D. Arnott and G. Pervan, A critical analysis of decision support systems research, Journal of Information Technology, 20, 2005, pp. 67-87.

[10] R. A. Hirschheim and H. K. Klein, Crisis in the IS field? A critical reflection on the state of the discipline, Journal of the Association for Information Systems, 4, 2003, pp. 237293.

[11]I. Benbasat and R. W. Zmud, Empirical research in information systems: the practice of relevance, MIS Q., 23, 1999, pp. 3-16.

[12]A. E. Rizzoli and W. J. Young, Delivering environmental decision support systems: software tools and techniques, Environmental Modelling \& Software, 12, 1997, pp. 237249.

[13]A. P. Wierzbicki and J. Wessels, The modern decision maker, In: J. Wessels, Modelbased decision support methodology with environmental applications, Kluwer Academic Publishers, 2000.

[14] M. Simo, L. Risto, A. Anna-Maria and J. Antti, Multi-criteria decision support in the liberalized energy market, Journal of Multi-Criteria Decision Analysis, 12, 2003, pp. 2742.

[15] R. M. Carlos and D. B. Khang, A lifecycle-based success framework for grid-connected biomass energy projects, Renewable Energy, 34, 2009, pp. 1195-1203. 